\title{
Review: ophthalmic non-steroidal anti-inflammatory drugs reduce pain without delaying healing in acute corneal abrasions
}

\author{
Weaver CS, Terrell KM. Evidence-based emergency medicine. Update: do ophthalmic nonsteroidal anti-inflammatory \\ drugs reduce the pain associated with simple corneal abrasion without delaying healing? Ann Emerg Med \\ 2003;41:134-40.
QUESTION: In patients with acute corneal abrasions, do ophthalmic non-steroidal anti-inflammatory drugs (NSAIDs) reduce pain without a delay in healing?

\section{Data sources}

Studies were identified by searching Medline (1966 to April 2002), the Cochrane Database of Systematic Reviews, ACP Journal Club, DARE, and the Cochrane Controlled Trials Registry and by reviewing bibliographies of relevant articles.

\section{Study selection}

Studies were selected if they were randomised controlled trials (RCTs) that compared ophthalmic NSAIDs with placebo, oral analgesic, or standard therapy in patients with acute corneal abrasions.

\section{Data extraction}

Data were extracted on sample size, study setting and quality, details of the intervention, and outcomes. The main outcomes included pain intensity (visual analogue scale or Numeric Pain Intensity Scale score) and time to healing.

\section{Main results}

5 RCTs (397 patients) met the selection criteria. Comparisons included ketorolac $0.5 \%$ with a "control vehicle" (1 RCT) or liquifilm tears (1 RCT), diclofenac $0.1 \%$ with normal saline solution (1 RCT) or natural tears (1 RCT), and indomethacin $0.1 \%$ plus gentamycin sulphate with gentamycin sulphate only (1 RCT). Reduction in pain intensity was greater in patients treated with ophthalmic NSAIDs than in those treated with control medications (5 RCTs) (table). The difference between groups in pain reduction was $\geq 13 \mathrm{~mm}$ on a $100 \mathrm{~mm}$ visual analogue scale (empirically perceived by patients to be a clinically important difference) in only 2 RCTs (table); however, in neither RCT did the 95\% CI around the difference exclude a value below this threshold. The groups did not differ for time to healing in 3 RCTs that reported the outcome.

For correspondence: Dr C S Weaver

Indiana University

School of Medicine,

Indianapolis, Indiana,

USA.

chsweave@iupui.edu

\section{Conclusion}

In patients with acute corneal abrasions, ophthalmic non-steroidal anti-inflammatory drugs reduce pain without a delay in healing.

\section{COMMENTARY}

Superficial corneal abrasions not associated with contact lens use generally resolve spontaneously without complications. They may cause disability due to pain during the first 24-48 hours following the injury, and it is because of pain that these patients are as likely to present to emergency rooms or other acute care settings as they are to ophthalmologists for treatment.

The review by Weaver $e t$ al of the evidence supporting the use of topical ophthalmic NSAIDS for corneal abrasions is an update on a previously published review. ${ }^{1}$ It closely follows the methodology of a systematic review but stops short of independent selection and assessment of studies and of statistical pooling of results. ${ }^{2}$ Weaver $e t$ al's well described search protocol did not include EMBASE/ Excerpta medica and, perhaps more importantly, did not attempt to locate unpublished proprietary studies that might exist and have observed negative results.

The 5 RCTs included in their review were done in ophthalmological and other acute care settings including emergency departments. 2 failed to report the use of analgesics other than the study medication, of which 1 also failed to report the absolute magnitude of the observed benefit. Although all 5 RCTs reported some degree of pain reduction within 24 hours of presentation, the clinical significance was unclear. Only 1 study reported an earlier return to work among patients receiving topical NSAIDS. Adverse effects were apparently minimal. The cost of these agents is variable and generic preparations exist. ${ }^{1}$ Until more definitive evidence is available, clinicians may choose to offer these agents primarily to patients for whom loss of a single day of work would be particularly inconvenient. Peter Wyer, MD, FACEP New York Presbyterian Hospital New York, New York, USA

1 Brown MD, Cordell WH, Gee AS. Ann Emerg Med 1999;34:526-34.

Wyer PC, Rowe BH, Guyatt GH, et al. Ann Emerg Med 2000;36:149-55

Ophthalmic non-steroidal anti-inflammatory drugs (NSAIDs) v placebo or standard therapy in acute corneal abrasions at 1-24 hours*

\begin{tabular}{|c|c|c|c|c|}
\hline Outcome & Comparisons (1 RCT each) & $\mathbf{n}$ & Scale & $\begin{array}{l}\text { Difference between groups }(95 \% \mathrm{Cl} \text { or } \\
\text { p value) } t\end{array}$ \\
\hline \multirow{5}{*}{$\begin{array}{l}\text { Mean reduction in pain } \\
\text { intensity from baseline }\end{array}$} & Ketorolac $0.5 \% v$ "control vehicle" & 100 & $0-10 \ddagger$ & $1.3(p=0.002) \S$ \\
\hline & Diclofenac $0.1 \% v$ normal saline & 40 & 0-100 mm VAS & $N R(p<0.05)$ \\
\hline & $\begin{array}{l}\text { Indomethacin } 0.1 \% \text { plus gentamycin sulphate } \\
v \text { gentamycin sulphate }\end{array}$ & 123 & $0-100 \mathrm{~mm}$ VAS & $5.9(p=0.007)$ \\
\hline & Ketorolac $0.5 \% v$ liquifilm tears & 85 & 0-50 mm VAS & $2(p<0.05)$ \\
\hline & Diclofenac $0.1 \% v$ natural tears & 49 & $0-100 \mathrm{~mm}$ NPIS & 21 (8 to 34$) \S$ \\
\hline
\end{tabular}

${ }^{*} \mathrm{RCT}=$ randomised controlled trial; VAS = visual analogue scale; NPIS = Numeric Pain Intensity Scale scores; NR = magnitude of effect not reported. Cl defined in glossary. †All significant differences favour NSAIDs.

t0 $=$ no pain and $10=$ severe pain

$\S$ Difference $\geq 13 \mathrm{~mm}$ on a $100 \mathrm{~mm}$ visual analogue scale (clinically important difference). 